\title{
Die Abbauzone Lagoinhos im römischen territorium metallorum Três Minas / Jales (Nordportugal)
}

Regula Wahl-Clerici, Markus Helfert, Thomas Schierl, João Moutinho, Vitor Gandra ${ }^{1}$

Haud aliter collis scrutator Hiberi, cum subiit longeque diem uitamque reliquit, si tremuit suspensus ager subitumque fragorem rupta dedit tellus, latet intus monte soluto obrutus, ac pentitus fractum obtritumque cadauer indignantem animam propriis non reddidit astris. acrior hoc Tydeus, animisque et pectore supra est.

Nicht anders der Prospekteur der iberischen Hügel, wenn er weit unter Tage gegangen ist und das Tageslicht sowie das Leben hinter sich gelassen hat, wenn das über ihm hängende Land bebt und die gerissene Erde ein plötzliches Krachen von sich gibt, verborgen tief drinnen im abgelösten Fels verschüttet ist, und dazu die völlig zerschmetterte und zerquetschte Leiche ihre empörte Seele nicht ihren eigenen Sternen zurückgibt.

PVBlivs PAPINIVs Stativs, Thebaid 6.880-885 (Übersetzung M. Scholz)

\section{Entdeckungsgeschichte}

Der römische Goldbergwerksdistrikt von Três Minas / Jales beeindruckt durch seine beiden großen Tagebaue und seine zur Erschließung, Förderung und Entwässerung notwendigen Tunnel, die stets mannshoch und teilweise mehr als $4 \mathrm{~m}$ breit aufgefahren wurden (Abb. 1) $)^{2}$. Weniger bekannt ist die kleinste Abbaustelle - in Anlehnung an die im Volksmund als „Lagos“= Seen bezeichneten großen Tagebaue mit dem Diminutiv „Lagoinhos“" genannt -, die sich im Streichen des anstehenden Schiefers von NW nach SE in östlicher Richtung anschließt. Lagoinhos bietet bei oberflächlicher Betrachtung ebenfalls das Bild eines zumindest im Westteil erfolgten Tagebaus (Abb. 2).

Anlässlich eines ersten Abstiegs 1992 auf die tiefer liegende Sohle $2(\mathrm{~N}-2)^{3}$ durch den Schacht 1 (P-1) wurde erkannt, dass der untertägige Bereich von Lagoinhos wesentlich größer ist, als die aktuell zugängliche Zone (Abb. 3 und 4). Leider konnten die Untersuchungen damals nicht weitergeführt werden. Erst 2015 und 2016 gelang es der Höhlenforschergruppe von „Alto Relevo, Clube de Montanhismo“ aus Valongo (Distrikt Porto/Portugal) aufgrund unserer Hinweise in die bislang unbekannten Abbaubereiche vorzustoßen (Abb. 5).

Im Folgenden werden erste Erkenntnisse zu Lagoinhos und zum dortigen Abbau bzw. den Abbaufortschritten vorgestellt. Sämtliche Schlussfolgerungen basieren auf den

\footnotetext{
${ }^{1}$ Weitere Mitarbeiter sind Dr. Annemarie Wiechowski und Patricia Machado. An den verschiedenen Begehungen von Lagoinhos nahmen folgende Mitglieder von „Alto Relevo Clube de Montanhismo“ teil: Augusto Monteiro, Beatriz Silva, Carlos Mendes, Eduardo Santos, Felisberto Vieira, Jorge Marques, Nélson Ribeiro, Vítor Rebelo.

${ }^{2}$ WAHL 1988; WAHL-CLERICI 2010; WAHL-CLERICI/HELFERT 2017.

${ }^{3}$ Sohle: Laufebene in einem bergmännischen Hohlraum (WAGNER ET AL 1985, 241).
} 
erhaltenen Spuren im obertägigen Bereich sowie auf den ersten durch die Begehung der Speläologen gewonnenen Daten im untertägigen Bereich. Es bleibt zu bemerken, dass die Abbauverhältnisse in Lagoinhos äußerst komplex waren. Zusätzlich entsteht infolge eines Einbruchs des Hangenden ${ }^{4}$ und des Einbrechens der südwestlichen Böschung im offenen Bereich ein unübersichtlicher Eindruck (Abb. 2 und 6). Ein ehemals mehrsöhliger Abbau und weitere Verstürze in der untertägigen Zone (Abb. 7), die teilweise wohl auch in jüngerer Zeit stattfanden, erschweren das Deuten der zahlreichen überlieferten Spuren zusätzlich. Mit aktuell zur Verfügung stehenden Methoden ist es nicht möglich, die Details zu erfassen, die für eine differenziertere Interpretation der erhaltenen Strukturen nötig wären, so dass wir uns auf eine überblicksartige Darstellung der bisherigen Beobachtungen beschränken müssen.

Grundsätzlich kann die Abbauzone Lagoinhos in einen offen daliegenden und in einen untertägigen Bereich eingeteilt werden. Ohne weitere Hilfsmittel ist die Mine auf einer Länge von rund $90 \mathrm{~m}$ und einer maximalen Breite von 8,30 m zugänglich (Abb. 8). Der erst 2015 entdeckte Abschnitt liegt etwas nach Nordosten versetzt größtenteils unterhalb der geschlossen erhaltenen Fortsetzung des offenen Bereiches (Abb. 4). Die gegeneinander verschobenen Teile der Lagerstätte zeigen etwas unterschiedliche Neigungen des schiefrigen Nebengesteins: Im oberen Teil sind die Schieferungsflächen mit $85^{\circ}$, im unteren Teil mit $70^{\circ}$ nach Nordosten geneigt (Abb. 2, 5).

\section{Der heute offen liegende Abbaubereich}

Spuren von Schlägel und Eisen sind in großen Teilen der nordöstlichen Böschung erhalten (Abb. 6). Meist können sie nicht bis zur Hangoberfläche verfolgt werden, was bedeutet, dass das Erz auch in diesem Bereich im Untertagebaubetrieb gewonnen wurde und somit das Hangende eingebrochen ist. $\mathrm{Ob}$ sich durch eine bereits geplante Aufnahme mittels 3D-Laserscanning nachweisen lässt, dass es mehrere Einstiegsschächte gab, werden weitere Untersuchungen zeigen ${ }^{5}$.

Abbildung 6 zeigt die Situation in der nordöstlichen Böschung. Links im Bild ist nach der ersten Felskante die Öffnung sichtbar, die durch das Schneiden des Abbaus mit einem älteren Prospektionsschacht entstand (Abb. 9). In der Fortsetzung nach rechts ist die Zone mit den Schlagspuren gut erkennbar. Ihre Begrenzung durch das ehemalige Hangende tritt klar hervor. In diesem Teil hat sich wohl ein kleiner Rest einer früheren Sohle erhalten, der sich in der Aufnahme als horizontal verlaufender Strich zeigt. Auffallend ist der große Felsabbruch, der sich ungefähr in der Mitte des Bildes befindet. Sowohl rechts wie auch unterhalb davon setzen sich die Arbeitsspuren fort. Die Frage, ob es sich hier um die Reste einer Verbindung resp. einer Stabilisierung zwischen zwei Abbauzonen handelt, kann beim aktuellen Stand der Untersuchungen nicht beantwortet werden.

Nur wenige Meter südöstlich schließt sich eine Übergangszone an, in der Schlagspuren fehlen. Danach beginnt der zweite Abbaubereich, in dem mehrere Sohlen nachgewiesen werden können.

\footnotetext{
${ }^{4}$ Hangende: Das über einer Vererzung befindliche Gebirge (EBD. 241).

${ }^{5}$ Domergue 2008, $101 \mathrm{f}$.
} 


\section{Der untertägige Abbaubereich}

Der Beginn dieser Abbauzone wird durch einen im Querschnitt länglichen Schacht 1 (P-1) markiert, der sich von der Tagesoberfläche trotz nachträglicher Veränderungen durch den Abbau und Einstürze bis auf eine Teufe von rund $28 \mathrm{~m}$ verfolgen lässt (Abb. 3 und 4). Besonders auffällig ist der südöstliche Stoß dieses Schachts mit seinen mehrfachen sorgfältig geschlagenen Erweiterungen (Abb. 10). Bis heute ist der Einstieg auf die tieferen Sohlen durch diesen Schacht möglich trotz des Bruchs, d.h. „des Einbruchs der Dach- bzw. Hangendenschichten in den bergmännischen Hohlraum“", der den Eindruck von Sohle 1 (N-1) stark verfälscht (Abb. 7).

Die Sohle $1(\mathrm{~N}-1)$ setzt sich geneigt noch über ca. $30 \mathrm{~m}$ weiter in den Berg hinein fort (Abb. 7, 8, 11). Bemerkenswert ist hier vor allem der nordöstliche Stoß, auf dem über eine Höhe von mind. $5 \mathrm{~m}$ Schlagspuren erhalten sind (Abb. 12). Die Abbauzone wurde auf verschiedenen Stufen aufgelassen. Abbildung 11 zeigt die höchste Stufe. Die Begrenzung nach Südosten wird durch die Probenentnahmestelle 174 markiert (Abb. 12). Hier ist bemerkenswert, dass ähnlich wie in Schacht 1 (P-1) (Abb. 11) der östliche Stoß abgestuft aufgelassen wurde. In der Folge können noch weitere, mehr oder weniger markante Abstufungen sowie die Begrenzung zwischen der Abbauzone und dem eingebrochenen südöstlichen Stoß identifiziert werden (Abb. 11, 12). Abbildung 13 zeigt eine der tieferen Auflassungen. Sie liegt ca. 3 m südöstlich. Das Ende der Abbauzone zeichnet sich durch zwei wiederum tiefer liegende Ortsbrüste ${ }^{7}$ auf verschiedenen Niveaus und drei Prospektionsstrecken (PR-2-4) ${ }^{8}$ zur weiteren Beprobung des Gebirges ${ }^{9}$ in alle Richtungen aus.

In der Gewinnzone ${ }^{10}$ auf der Sohle (N-1), die vom heute obertägig liegenden Bereich aus zugänglich ist, konzentrieren sich Stellen von Probenentnahmen des staatlichen portugiesischen Serviço de Fomento Mineiro (heute: Instituto Geologico e Mineiro). Dieses Amt führte in den 80er Jahren des letzten Jahrhunderts gründliche Prospektionsarbeiten durch. Sie haben ihre Spuren in Form der rot umrandeten Vierecke um die Probenentnahmestellen bis heute hinterlassen. Für Lagoinhos sind uns auch die dabei gewonnenen Ergebnisse bekannt. Der Plan (Abb. 8) zeigt, dass mit einer Ausnahme sämtliche goldhaltigen Proben aus der ehemaligen Abbauzone stammen, wobei die Geologen keinen Unterschied zwischen eigentlichen Abbaubereichen und Bruchzonen machten.

\footnotetext{
${ }^{6}$ WAGNER ET AL 1985, 240.

${ }^{7}$ Ort/Ortsbrust: Arbeitsplatz der Bergleute während des Streckenvortriebs, besonders das jeweilige Ende eines Grubenbaus im Gestein bei Herstellung und Betrieb (EBD. 241).

${ }^{8}$ Strecke: Im Gestein oder Erz mehr oder weniger horizontal aufgefahrener Grubenbau (EBD. 242).

${ }^{9}$ Gebirge: Das eine Lagerstätte umgebende Gestein (EBD. 241).

${ }^{10}$ Gewinnung: Herauslösen des Erzes aus dem Gebirgsverband durch bergmännischen Betrieb (EBD. 241).
} 


\begin{tabular}{|l|l|l|l|l|l|l|l|l|l|l|}
\hline \multicolumn{2}{|l|}{ Probenentnahmestellen mit Gold- und Silbergehalten } \\
\hline $\begin{array}{l}\text { Proben- } \\
\text { nummer }\end{array}$ & 146 & 147 & 150 & 155 & 156 & 160 & 161 & 165 & 173 & 174 \\
\hline $\begin{array}{l}\text { Länge in } \\
\mathrm{m}\end{array}$ & 0,3 & 0,4 & 0,45 & 0,45 & 0,35 & 0,55 & 0,35 & 0,5 & 0,3 & 0,7 \\
\hline $\begin{array}{l}\text { Gehalt } \\
\text { g/T Au }\end{array}$ & 1,4 & 27,7 & 3,2 & 0,8 & 1,8 & 0,9 & 3,6 & 1,9 & 1,6 & 12,3 \\
\hline $\begin{array}{l}\text { Gehalt } \\
\text { g/T Ag }\end{array}$ & 0,2 & 6,8 & 2,1 & 3,9 & 2,3 & 3,5 & 4,4 & 0,9 & 2,7 & 3,8 \\
\hline
\end{tabular}

Die Goldgehalte sind im Durchschnitt gering, so dass ein antikes Auflassen des Abbaus nachvollziehbar ist. Bei den beiden isolierten Proben mit den relativ hohen Gehalten (Probennummern 147: 22,7 g/T und 174: 12,3 g/T) machen sich wahrscheinlich der sogenannte Nugget-Effekt sowie die sehr unregelmäßige Verteilung des Goldes in der Lagerstätte bemerkbar. Das heißt, die analysierten Proben sind nicht repräsentativ, und zufällig erfasste Goldkörnchen können das Bild verzerren ${ }^{11}$. Diese Problematik war den Römern offensichtlich bekannt, denn der Abbau wurde eingestellt. Es bleibt zu ergänzen, dass in fast allen Proben Silbergehalte festgestellt wurden, die allerdings unterhalb einer abbauwürdigen Konzentration in primären Lagerstätten mit den aufwendigen Aufbereitungsprozessen liegen ${ }^{12}$. Die Silbergehalte sind meist ans Gold gebunden. Gold in Três Minas enthält bis zu 25\% Silber.

Besonders komplex sind die Verhältnisse im Bereich der nur durch den Schacht 1 (P1) zugänglichen tieferen Sohlen 2, 3 und 4 (N-2, N-3, N-4) (Abb. 4. 11). Der heute große Hohlraum entstand durch das Nachreißen ${ }^{13}$ eines von oben her begonnenen Abbaus. Bemerkenswert ist auch die durch die Begehung des östlichen Bereichs auf Sohle 3 (N-3) gewonnene Erkenntnis, dass es mindestens noch einen weiteren Zugang durch einen Blindschacht (P-3?) zwischen den Sohlen 1 und 2 (N-1, N-2) gab, wobei letzterer in diesem Bereich auch nicht in Spuren erhalten ist ${ }^{14}$. Die Reste einer Prospektionsstrecke (Pr-6) sowie eine Abstufung im südöstlichen Stoß ${ }^{15}$ sind Hinweise auf die Fortsetzung der ehemaligen Sohle (N-3) (Abb. 5) und belegen das frühere Abbauniveau, das im südöstlichsten Teil noch erhalten ist (N-3). Am südöstlichen Ende zeichnen sich Spuren eines Schachts (P-4?) ab.

Die tiefste Sohle 4 (N-4) ist stark verschlammt und liegt teilweise unter dem Niveau des Wassers, das sich hier sammelt, da es offensichtlich keinen Abfluss hat, sondern nur durch die kleinsten Spalten im anstehenden Schiefer entweichen kann (Abb. 9) ${ }^{16}$.

\footnotetext{
${ }^{11}$ Wir danken Dr. A. Wiechowski, RWTH Aachen, für die freundliche Auskunft.

12 BACHMANN 1993; WAHL-CLERICI ET AL. 2012a; WAHL-CLERICI/WIECHOWSKI 2012; WAHLCLERICI/HELFERT 2017.

${ }^{13}$ Nachreißen: Einen bergbaulichen Hohlraum erweitern (WAGNER ET AL 1985, 241).

${ }^{14}$ Blindschacht: Ein nicht zu Tage gehender senkrechter Verbindungsschacht innerhalb der Grube, der zwei oder mehrere Sohlen miteinander verbindet (EBD. 240).

${ }^{15}$ Stoß: Seitenwand eines bergmännischen Hohlraums (EBD. 242).

${ }^{16}$ Der Einstieg der Mitglieder der Gruppe Alto Relevo erfolgte im September 2015 sowie im Oktober 2016.
} 
Die gepunkteten Linien im Übersichtsplan (Abb. 4) zeigen mögliche Abbauzonen an, wobei der Verdacht, dass sich die beiden Einstiegszonen Schacht 1 (P-1) und Schacht 2 (P-2) untertägig treffen, nicht von der Hand zu weisen ist.

Mit Hilfe der gut ausgebauten Prospektionsstrecke PR-5 (Abb. 15) wurde das Gebirge in nordöstlicher Richtung exploriert.

Die nördlich der Abbauzone angehäuften Halden sind im Verhältnis zu denjenigen der Tagebaue Corta de Covas und Corta da Ribeirinha auffallend klein (Abb. 16). Das könnte ein Anzeichen dafür sein, dass große Teile des Abbaus aufbereitet werden konnten. Ungeklärt ist bislang die Frage, ob möglicherweise ein Abbau mit Versatz erschlossen werden kann. Das bedeutet, steriles Material wurde zum Ausfüllen eines ausgehauenen Raumes angehäuft ${ }^{17}$. Voraussetzung dafür wäre ein teilweiser Abbau von unten, d.h. der Abraum dient als Versatz der abgebauten Strecke und zusätzlich als Sohle für den weiteren und in diesem Fall höher liegenden Abbau. Grundsätzlich kann diese Möglichkeit nicht ausgeschlossen werden.

Auffällig ist auch das Fehlen von Pochsohlen und Erzmühlen resp. von entsprechenden Fragmenten bei Lagoinhos, wie sie in den anderen Abbaubereichen und dabei angeschlossenen Aufbereitungszonen in Massen gefunden werden. Auch wenn der größte Teil der Pochsohlen und Erzmühlen wohl nach Ribeirinha abtransportiert wurde, kann davon ausgegangen werden, dass immer noch ein paar Fragmente zurück geblieben wären. Somit müssen wir festhalten, dass bislang unbekannt ist, wo das Fördergut aus Lagoinhos aufbereitet wurde ${ }^{18}$.

\section{Untertagebau in römischer Zeit auf der iberischen Halbinsel}

Die summarische Beschreibung der Abbauverhältnisse in Lagoinhos belegt, dass es sich um eine rein untertägige Gewinnung handelte. Damit hebt sich die Abbauzone deutlich von den beiden großen Tagebauen, Corta de Covas und Corta da Ribeirinha, sowie von der Förderung in den langgezogenen Erzgängen von Campo de Jales und Gralheira ab, die beide ebenfalls obertägig abgebaut wurden (Abb. 17, 18) ${ }^{19}$.

Domergue bespricht in seiner umfassenden Übersicht zum antiken Bergbau auf der iberischen Halbinsel auch den Untertagebau ${ }^{20}$. Grundsätzlich hält er fest, dass der Abbau wie seit eh und je der Vererzung folgte und, wenn immer möglich, auf diese beschränkt wurde. Die von ihm postulierte Methode des untertägigen Feuersetzens, zu der er kein Beispiel anführt, konnte beim Abbau in der Serra de Santa Justa bei Valongo (Concelho Valongo/P) nachgewiesen werden ${ }^{21}$. Feuersetzen war in Três Minas eine oft angewandte Technik, die allerdings nur obertägig eingesetzt wurde bzw. nur dort nachweisbar ist ${ }^{22}$.

\footnotetext{
17 WAGNER ET AL 1985, 242.

${ }^{18}$ WAHL-CleriCi ET AL. 2012a; WAHL-CLERICI/HELFERT 2017; WAHL-CLERICI (in Vorbereitung).

${ }^{19}$ Domergue 1990, 428 zählt die Mine von Campo de Jales fälschlicherweise zu den Untertagebauen.

${ }^{20}$ EBD. 413-462.

${ }^{21}$ EBD. 428.

22 WAHL 1997.
} 
In der von Domergue publizierten Liste zum Untertagebau finden sich leider keine Angaben zur Chronologie der fraglichen Bergwerke auf der Pyrenäenhalbinsel ${ }^{23}$. Entsprechend der römischen Eroberungsphasen setzte der Abbau im Süden und Südosten bereits in republikanischer Zeit ein, nachdem die Erzgewinnung auf der iberischen Halbinsel schon auf eine lange Tradition zurückblicken konnte ${ }^{24}$. Unberücksichtigt bleiben in der Liste von Domergue die topographischen und geologisch-mineralogischen Situationen ${ }^{25}$. Ebenso wenig ist es ihm möglich, die jeweilige Organisationsform des Abbaus, direkt dem Fiskus unterstellt oder vom Fiskus in Form von Pachten ausgegeben, zu berücksichtigen, so dass die von ihm angeführten jeweils erreichten Teufen zwar beeindrucken, aber für einen Vergleich weitgehend ungeeignet sind ${ }^{26}$. Immerhin belegen die Mine von Castripicón (Co 43. Provinz Córdoba/E), die eine Teufe von $50 \mathrm{~m}$ mit drei Abbausohlen aufweist, und diejenige von Calo (H 16, Provinz Huelva/E), die eine Teufe von $190 \mathrm{~m}$ mit sieben nicht ganz eindeutigen Abbausohlen hatte, dass es bei sorgfältiger Aufnahme möglich ist, die Abbaufortschritte nachzuvollziehen ${ }^{27}$. Ebenso bedeutsam ist der Untertagebau in der Serra de Santa Justa bei Valongo, der durch die mühsamen Reinigungsarbeiten von Alto Relevo der Forschung vor wenigen Jahren überhaupt erst zugänglich gemacht wurde ${ }^{28}$.

Von grundsätzlicher Bedeutung für den Abbau in römischer Zeit sind die möglichen Organisationsformen ${ }^{29}$. Die Goldminen im Nordwesten der iberischen Halbinsel waren stets der fiskalischen Verwaltung untergeordnet, d.h. ein kaiserlicher Prokurator stand dem Bergwerk vor. Sämtlicher Gewinn floss direkt in die kaiserliche Kasse. Aus den schriftlichen Quellen aus dem 2. Jahrhundert n. Chr. von Vipasca (Aljustrel, Concelho Castro Verde/P) und von Alburnus Maior (Rosia Montana/RO) ist bekannt, dass dort vom Repräsentanten des Fiskus Pachten vergeben wurden, der den Gewinn in Form von Zinsen einnahm. Dank der Untersuchungen von Cauuet an diesen beiden Orten und der Darstellung der Abbaufortschritte in der Corta de Covas von Três Minas durch Wahl-Clerici wissen wir, dass die Abbauzonen die Organisationsform spiegeln ${ }^{30}$.

Damit stellt sich die Frage, ob trotz der Unvollständigkeit der Untersuchung auch für Lagoinhos entsprechende Aussagen möglich sind. Gibt es Hinweise für einen Abbau in Pachtform oder müssen wir davon ausgehen, dass auch hier die direkte staatliche Organisation das Zepter führte? Es würde wohl dem Forschungsstand und den Verhältnissen vor Ort nicht gerecht, wenn wir die drei heute grosso modo unterscheidbaren Abbauzonen als Pachten werten würden.

Zur Chronologie des Abbaus in Lagoinhos sind aktuell keine konkreten Aussagen möglich, da jegliche Art von Funden fehlt. Somit müssen wir uns nur mit den

\footnotetext{
${ }^{23}$ Domergue 1990, 430-432.

24 EBD. 83-173.

${ }^{25}$ EBD. 430-432.

${ }^{26}$ Die größten von EBD. 430-433 angeführten Teufen erreichten die Minen von Mazarrón (Mu10. Ag, Pb. Provinz Murcia/E) mit 340 m und Cabezo Rajado (Mu4. Ag, Pb. Provinz Murcia/E) mit 280 m, d.h. in beiden Minen wurde unter dem Meeresniveau gearbeitet. - Zum Bergbau als Spiegel der Organisationsform vgl. DOMERGUE 2008, 179-213; WAHL-CLERICI/HELFERT 2017; HIRT 2010 passim.

${ }^{27}$ Domergue 1990, 428.

${ }^{28}$ EBD. 430.

${ }^{29}$ DOMERGUe 2008, 196.

${ }^{30}$ Abbildung von B. Cauuet in Domergue 2008, 205 Abb. 124; WAHL-Clerici 2010; WAHLCLERICI/HELFERT 2017.
} 
allgemeinen Angaben zu den Betriebszeiten des Bergbaus im territorium metallorum Três Minas / Jales begnügen, die sich über fast das gesamte 1. und 2. Jahrhundert n. Chr. erstrecken. $\mathrm{Ob}$ die erhaltenen Monumente von Lagoinhos genug Hinweise liefern, den Abbau in eine besonders frühe Phase zu platzieren, kann beim aktuellen Stand der Forschung nicht geklärt werden.

Es ist auch darauf hinzuweisen, dass im Bereich der Abbauzone von Lagoinhos eine auffällige Häufung von Prospektionsbauten beobachtet werden kann. Das belegt, dass die dortige Lagerstätte gründlich und in alle Richtungen beprobt wurde, auch wenn sich die abbauwürdigen Gehalte im Wesentlichen auf eine lange und eher schmale Zone von bislang noch unbekannter Tiefe beschränken ${ }^{31}$.

\section{Ergebnisse}

Diese nur sehr kurze Übersicht der neu gewonnenen Ergebnisse in Lagoinhos erweitert unsere Kenntnis zum römischen Bergbau im territorium metallorum Três Minas / Jales. Alles deutet darauf hin, dass es sich bei Lagoinhos einstmals um einen reinen Untertagebau handelte, dessen Einstieg von der Hangoberfläche her durch den breiten Schacht 1 gewährleistet war. Die Lagerstätte folgte exakt dem Streichen des Schiefers, was sich in der Lage und Ausrichtung der beiden Tagebaue „Corta de Covas“, „Corta de Ribeirinha“" und von „Lagoinhos“ widerspiegelt (Abb. 1).

Der langgezogene, aber stets relativ schmale Abbau erinnert an die Verhältnisse in der Lagerstätte Gralheira, einem Erzgang, der sich über eine Länge von ca. 5 km erstreckt, wobei er sich teilweise in mehrere Stränge auffächert (Abb. 17). Alles weist darauf hin, dass die Gewinnung im Erzgang von Gralheira ausschließlich im Tagebauverfahren erfolgte $^{32}$.

Aus Lagoinhos gibt es im Gegensatz zu den Lampenfunden in der Galeria do Pilar resp. der Galeria do Texugo keine Hinweise auf den vermutlichen Zeitraum der Ausbeutung. Bergbauliche Monumente untertägigen Abbaus in römischer Zeit sind im Nordwesten der iberischen Halbinsel selten und beruhen teilweise auf einer falschen Interpretation der Gegebenheiten ${ }^{33}$. Parallelen finden sich u. U. in Valongo, wo in der römischen Kaiserzeit ebenfalls Gold aus primären Lagerstätten gewonnen wurde.

Verschiedene Beobachtungen und Indizien lassen vermuten, dass der Vortrieb in Três Minas auch an anderen Stellen untertägig erfolgte. Darauf weisen die Spuren des Grubenunglücks in einem tonnlägigen Schacht, der im hinteren Bereich der Galeria dos Alargamentos abzweigt und vermutlich der Prospektion tiefer gelegener Zonen diente. Nachweislich rutschten während des Auffahrens oder der Nutzung dieses Treppenschachts zwei ca. $5 \mathrm{~m}$ auseinanderliegende Teile um 2,20 m resp. 3,60 m in die Tiefe. In den erst durch das Absacken freigelegten Felswänden, die seit dem Ereignis den Zugang erschweren bzw. verhindern, befinden sich nachträglich eingearbeitet Lampennischen, die den Rettungshelfern zur Position der Beleuchtung für die auf das Unglück folgende Rettungsaktion eingeschlagen wurden. Entscheidend in unserem Zusammenhang ist, dass sich unterhalb der Bruchzone sehr wahrscheinlich ein

\footnotetext{
${ }^{31}$ WAHL-CLERICI/WIECHOWSKI 2013.

32 WAHL 1988; WAHL 1998; WAHL-CLERICI/WIECHOWSKI 2013.

${ }^{33}$ Bei der Cueva de Juan Rata in der Abbauzone von Alto del Palo (O5. Provinz Asturien/E) handelt es sich vermutlich um einen Prospektionsstollen (DOMERGUE 1987, 422-423).
} 
Hohlraum befand, denn sonst wäre ein plötzliches Einbrechen von Gesteinsmassen in diesem alten Gebirge aus kristallinem Schiefer nicht möglich gewesen ${ }^{34}$.

Die vorläufig noch summarische Übersicht über die in Lagoinhos erhaltenen Spuren lässt in den Grundzügen das Vorgehen der Römer beim Abbau erkennen. Den antiken Bergleuten gelang es offensichtlich, die Erzgewinnung auf diejenigen Zonen zu beschränken, in denen abbauwürdige Goldgehalte lagerten. Es scheint, dass ein entsprechendes Vorgehen hier möglich war, ganz im Gegensatz zu den weiter nordwestlich gelegenen Lagerstättenbereichen, wo auch größere Volumen von taubem Gestein abgetragen werden mussten, um an das Gold zu gelangen. Aufschluss über die Fortschritte der Erzgewinnung in Lagoinhos wird aber erst eine komplette 3DAufnahme liefern.

Der eingangs zitierte Text des römischen Dichters Statius könnte den Einbruch des Hangenden in der Abbaustelle Lagoinhos und die Auswirkungen für die Bergleute beschreiben. Denn auch ein Einbrechen großer Felsmassen verursacht gelegentlich ein sogenanntes Einsturzbeben, bei dem seismische Gründe als Ursache ausgeschlossen werden dürfen.

\section{Abgekürzt zitierte Literatur}

BACHMANN 1993

DOMERGUE 1987

DOMERGUE 1990

DOMERGUE 2008
H.-G. BACHMANN, Zur Metallurgie der römischen Goldgewinnung in Três Minas und Campo de Jales in Nordportugal. In: H. Steuer/U. Zimmermann (Hrsg.), Montanarchäologie in Europa. Berichte zum internationalen Kolloquium „Frühe Erzgewinnung und Verhüttung in Europa“ in Freiburg/Breisgau vom 4. bis 7. Oktober 1990. Archäologie und Geschichte - Freiburger Forschungen zum ersten Jahrtausend in Südwestdeutschland 4 (Sigmaringen 1993) 153160.

C. Domergue, Catalogue des mines et des fonderies antiques de la Péninsule Ibérique. Publications de la Casa de Velázquez Série Archéologie 8 (Madrid 1987) I und II.

C. Domergue, Les mines de la Péninsule Ibérique dans l'antiquité romaine. Collection de l'École Française de Rome 127 (Rome 1990).

C. Domergue, Les mines antiques. La production des métaux aux époques grecque et romaine. Antiqua (Paris 2008).

\footnotetext{
${ }^{34}$ WAHL-CLERICI ET AL. 2012b.
} 
HIRT 2010

WAHL 1988

WAHL 1998

WAHL-CLERICI 2010

WAHL-CLERICI (in Vorbereitung)

WAHL-CLERICI ET AL. 2012a

WAHL-CLERICI ET AL. 2012b
A. M. HIRT, Imperial Mines and Quarries in the Roman World. Organizational Aspects 27 BC-AD 235 (New York 2010).

J. WAHL, Três minas. Vorbericht über die archäologischen Untersuchungen im Bereich des Römischen Goldbergwerks 1986/87. Madrider Mitteilungen 29, 1988, 221-244.

J. Wahl, Aspectos tecnológicos da indústria mineira e metalúrgica romana de Três Minas e Campo de Jales (Concelho de Vila Pouca de Aguiar). In: Actas do Seminário Museologiea e Arqueologia Mineiras. Publicações do Museu do Instituto Geologico e Mineiro (Lisboa 1998) 5768.

R. WAHL-CLERICI, Untersuchungen zum Abbaufortschritt in der Corta de Covas (Tagebau A) im römischen Goldbergwerk von Três Minas (conc. Vila Pouca de Aguiar, Portugal). In: J. G. Gorges/T. Nogales Basarrate (Hrsg.), Naissance de la Lusitanie romaine (Ier av. - Ier ap. J.-C.). VIIe Table ronde internationale sur la Lusitanie romaine (Toulouse 8-9 novembre 2007) $=$ Origen de la Lusitania romana (siglos I a.C. - I d.C.): VII Mesa Redonda Internacional sobre la Lusitania Romana (Toulouse 8-9 novembre 2007) (Toulouse/Mérida 2010) 437-458.

Territorium metallorum Três Minas / Jales. Eine Übersicht über die Forschungen (in Vorbereitung).

R. WAHL-Clerici/A. WIECHOWSKI/M. HELFERT/B. RAMMINGER, Die Golderzaufbereitung im römischen Bergwerks von Três Minas und Campo de Jales in Nordportugal. Der Anschnitt 64, 2-3, 2012, 109118.

R. WAHL-CLERICI/M. HELFERT/A. WIECHOWSKI, Três Minas - Eine Bergwerkskatastrophe mit Rettungsaktion in der römischen Kaiserzeit. Frankfurter elektronische Rundschau zur Altertumskunde 17, 2012, 12-28.

Wahl-Clerici/Wiechowski 2012 R. Wahl-Clerici/A. Wiechowski, Der „Forno dos Mouros“. Ein Aufbereitungs- und Verhüttungsplatz im römischen Goldbergwerksdistrikt von Três Minas und Campo de Jales. In: H. Lasch/B. Ramminger (Hrsg.), Hunde, Menschen, Artefakte. Gedenkschrift für Gretel Callesen. Internationale 
Archäologie. Studia honoraria 32 (Rahden/Westfalen 2012) 325-337.

WAHL-Clerici/WIECHOWSKi 2013 R. WAHL-Clerici/A. WIECHOWSKI, Untersuchungen zur antiken Prospektion von Erzlagerstätten mit bergbaulichen Beispielen aus dem römischen Goldbergwerksdistrikt von Três Minas, Gralheira und Campo de Jales in Nordportugal. Madrider Mitteilungen 54, 2013, 299-326.

Wahl-Clerici/Helfert 2017 R. Wahl-Clerici/M. Helfert, A indústria mineira do territorium metallorum romano de Tresminas e Campo de Jales. In: C.M. Braz Martins/P. Machado/J. I. Ferrão de Paiva Martins (Hrsg.), Territorium Metallorum Tresminas/Jales, Vila Pouca de Aguiar. I Simposio Internacional (Vila Pouca de Aguiar 2017) 47-94.

WAGNER ET AL. 1985

G. A. Wagner/G. Weisgerber/W. Kroker (Hrsg.), Silber, Blei und Gold auf Sifnos: Prähistorische und antike Metallproduktion. Der Anschnitt, Beiheft 3. Veröffentlichungen aus dem Deutschen Bergbau-Museum Bochum 31 (Bochum 1985) 240-242.

\section{Adressen der Autoren}

Lic. Phil. Regula Wahl-Clerici

Email: regulawahl@gmail.com

Dr. Markus Helfert

Email: m.helfert@em.uni-frankfurt.de

Thomas Schierl M. A.

Email: thomasschierl@gmx.de

João Moutinho

Email: jnm@fe.up.pt

Vitor Gandra

Email: vitorgandra@gmail.com 


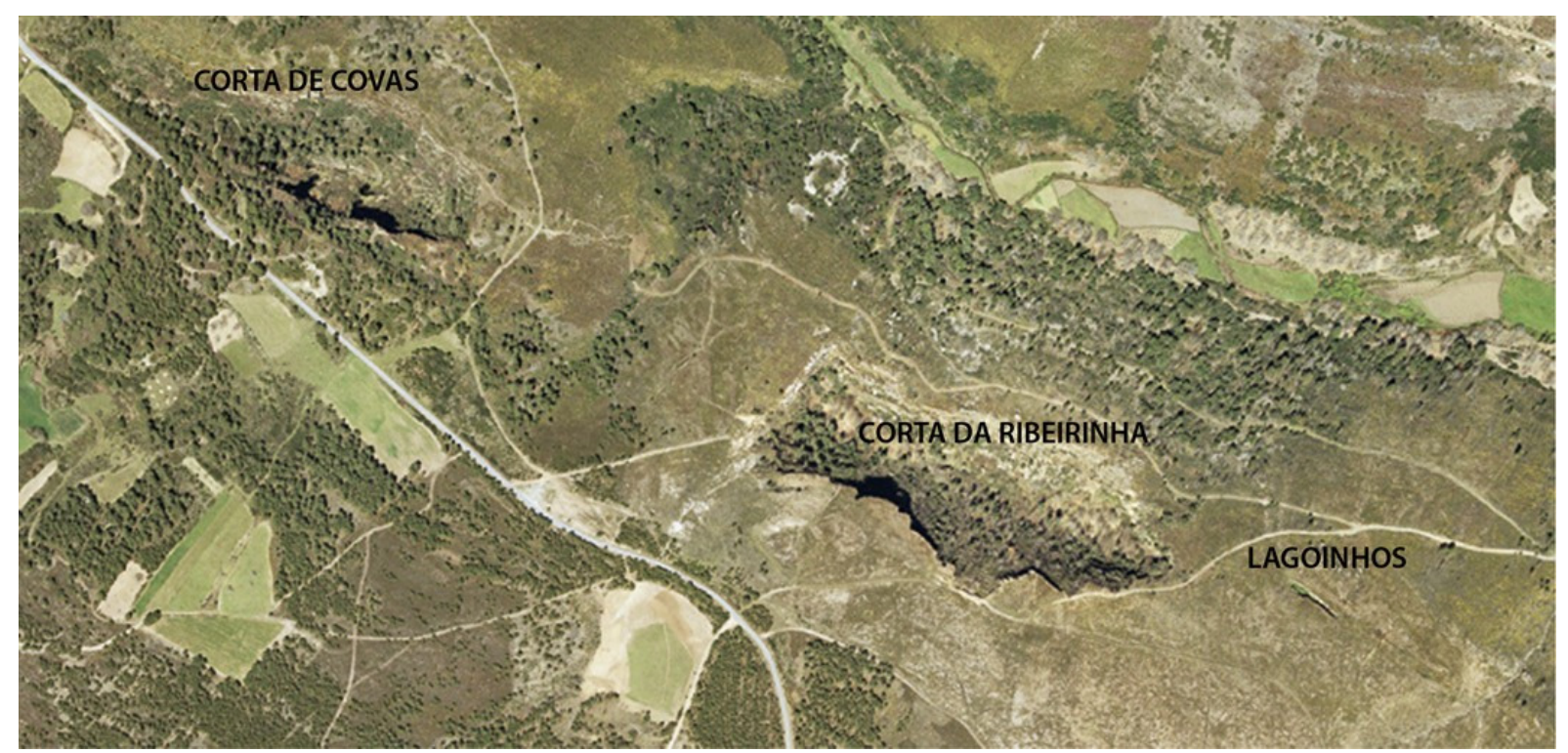

Abb. 1. Abbauzone Três Minas mit dem Untertagebau Lagoinhos und den beiden Tagebauen Corta de Covas und Corta da Ribeirinha. Vorlage Ortofotomapas 1:5000 CM Vila Pouca de Aguiar. Gestaltung R. Wahl-Clerici.

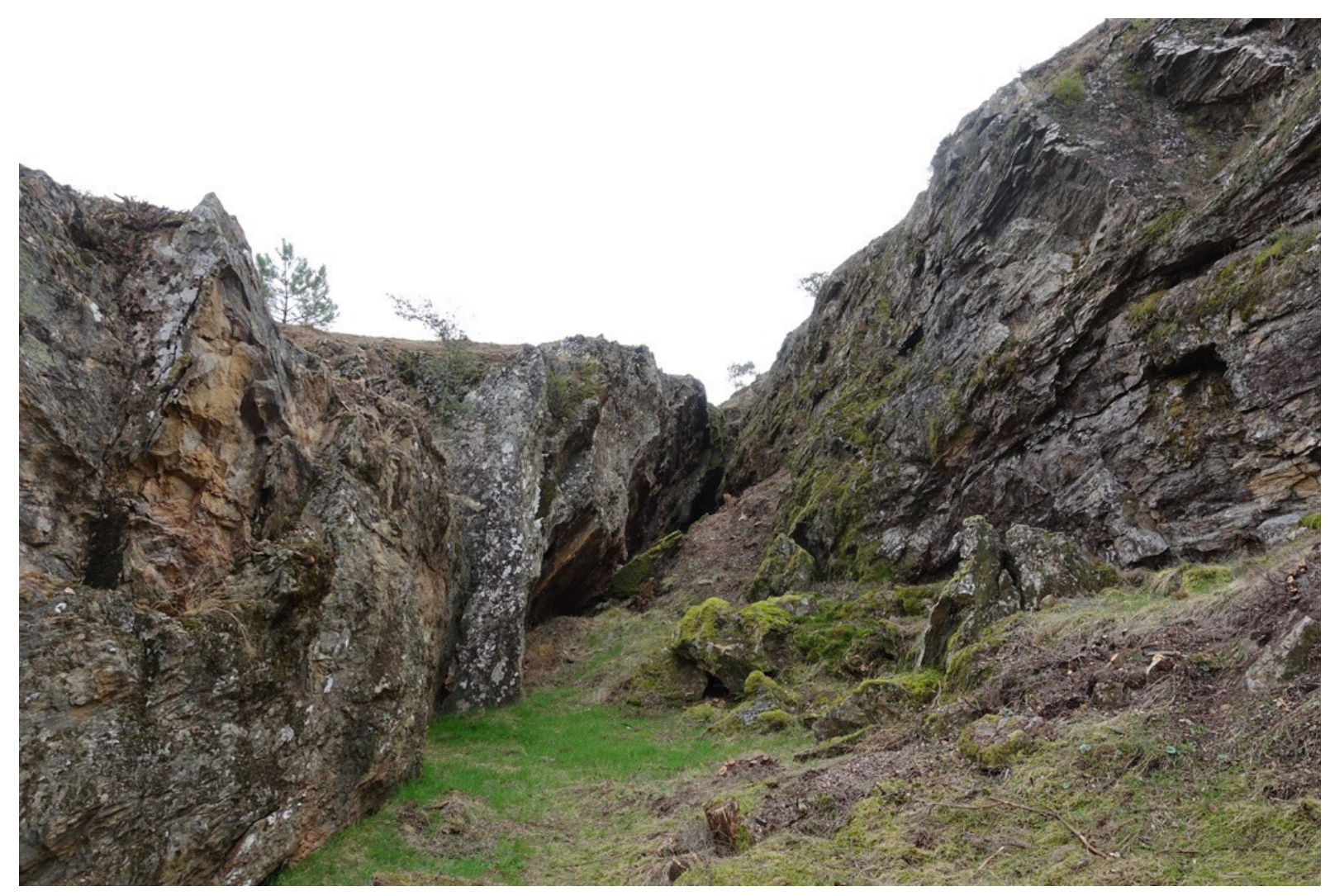

Abb. 2. Heute offen liegender Bereich von Lagoinhos. Von Nordwesten. Links die abgearbeitete Wand, rechts der verstürzte Bereich. Foto R. Wahl-Clerici. 
Frankfurter elektronische Rundschau zur Altertumskunde 33 (2017)

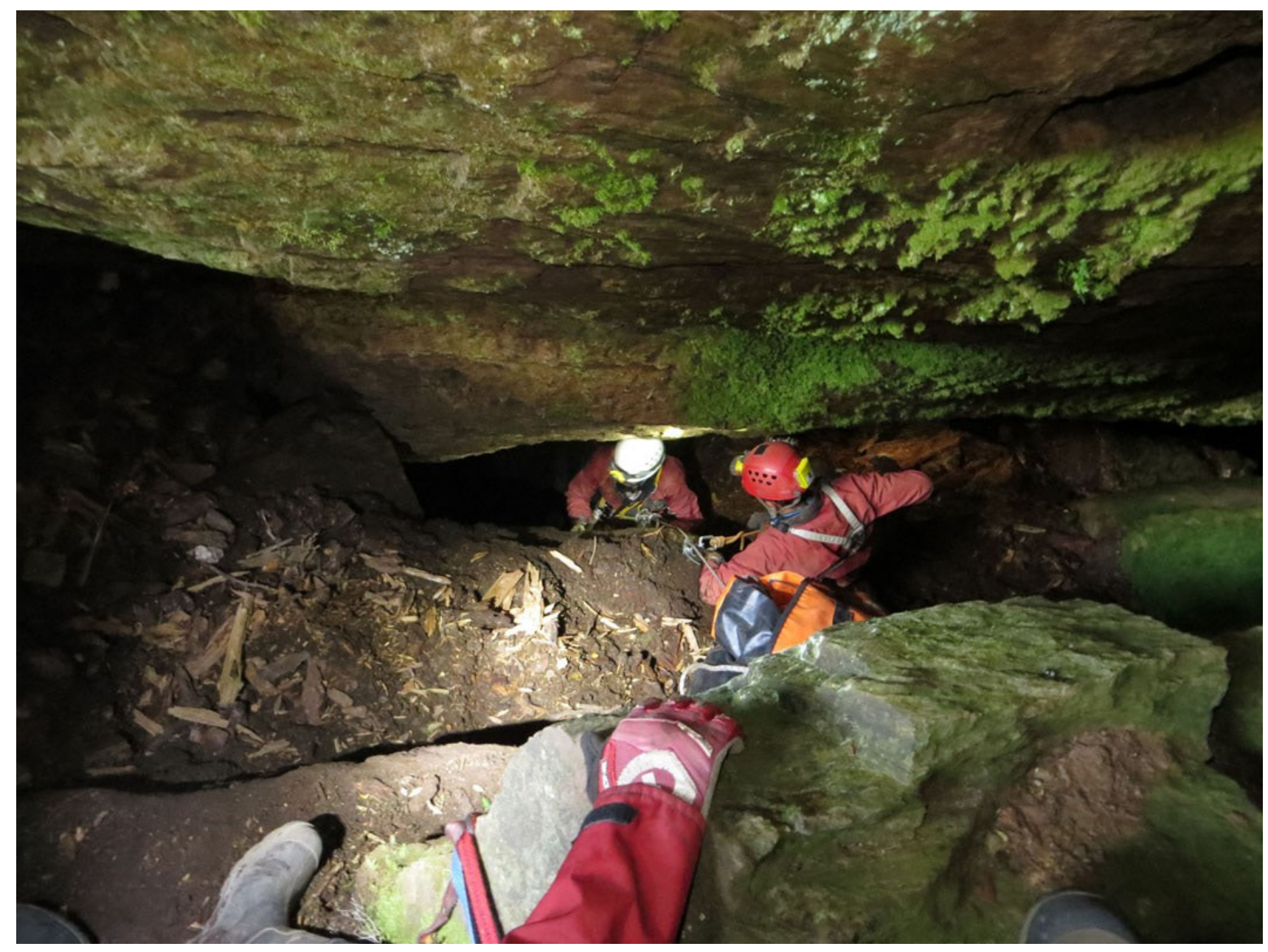

Abb. 3. Einstieg durch den Schacht P-1 in den Untertagebau. Foto R. Wahl-Clerici. 
Frankfurter elektronische Rundschau zur Altertumskunde 33 (2017)
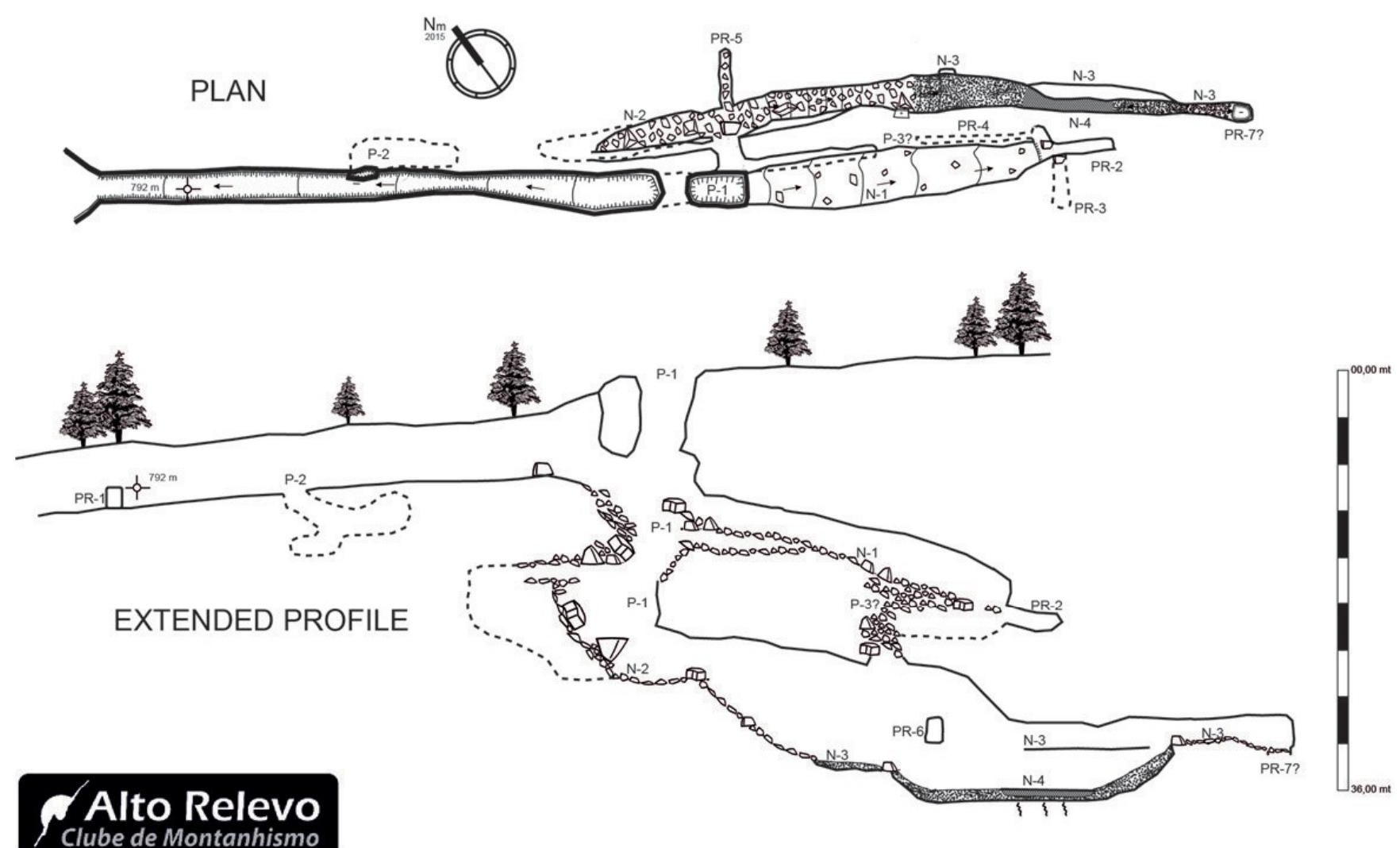

Clube de Montanhismo

www.altorelevo.org

Corta de Lagoinhos

N 41,493278 W-7,515167 (WGS84)

Vila Pouca de Aguiar - Portugal

Cavity ID: id292

Development: $121 \mathrm{~m}$

Topographic team: Carlos Mendes, Hugo Santos, João Moutinho, Luis Sousa, Vitor Gandra

Topography date: 30 Ago 2015

Equipment: Disto $\mathrm{A} 3(\mathrm{x})$ Leica

Final drawings: Vitor Gandra

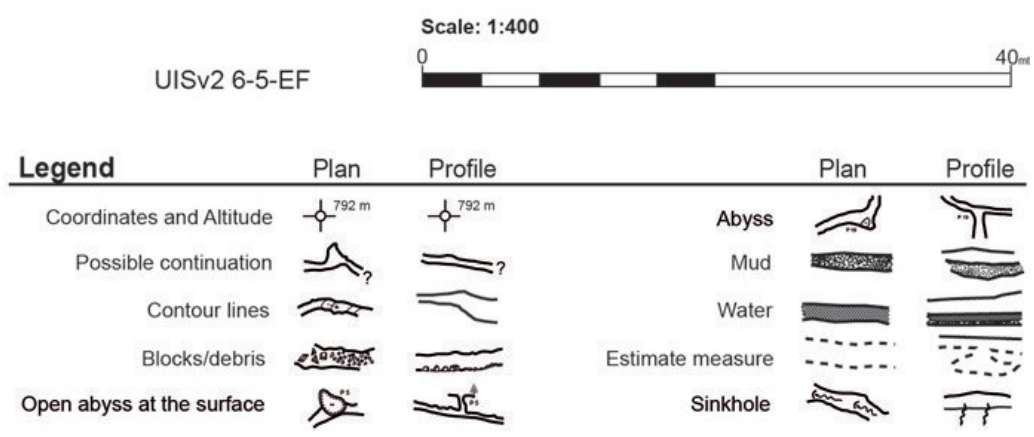

Abb. 4. Schematisierter Grundriss und Schnitt von Lagoinhos. Aufgrund der Aufnahmetechnik mit dem Disto A3(x) Leica ergibt sich die leichte Abweichung der Länge von Grundriss und Schnitt. P = Schächte, PR = Prospektionen,

$$
\mathrm{N}=\text { Sohlen. }
$$




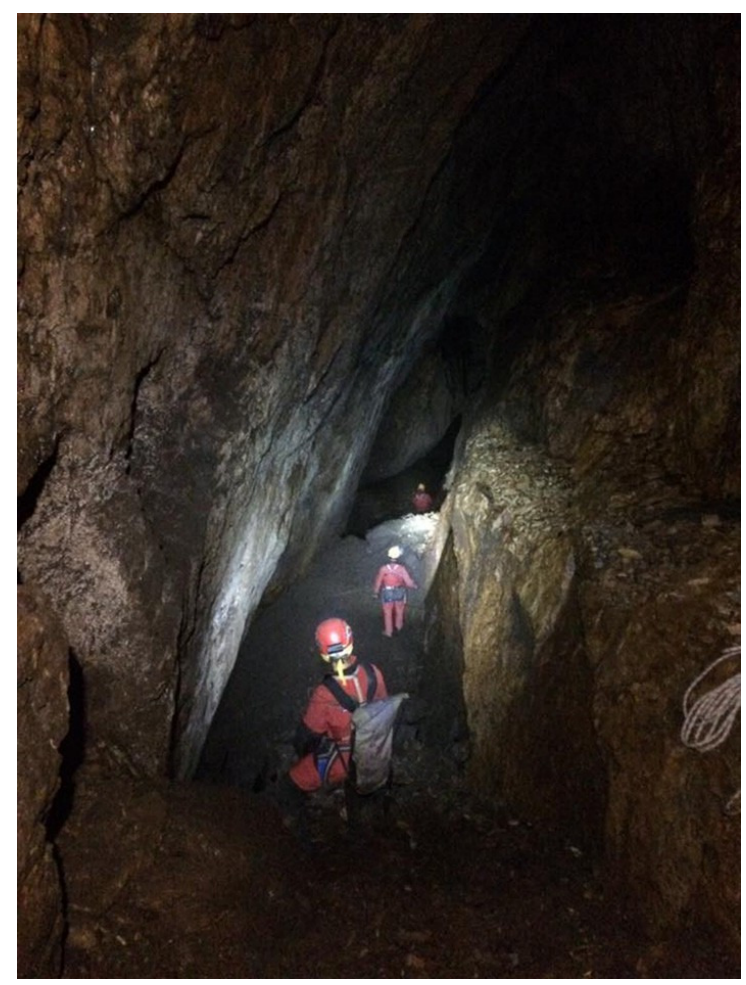

Abb. 5. Blick von Sohle N-2 in Richtung Südost auf die Sohlen N-3 und N-4. Rechts im Bild sind der im südwestlichen Stoss erhaltene Rest der Sohle N-2 sowie schwach der Ansatz der Prospektionsstrecke PR-6 erkennbar. Foto V. Gandra.

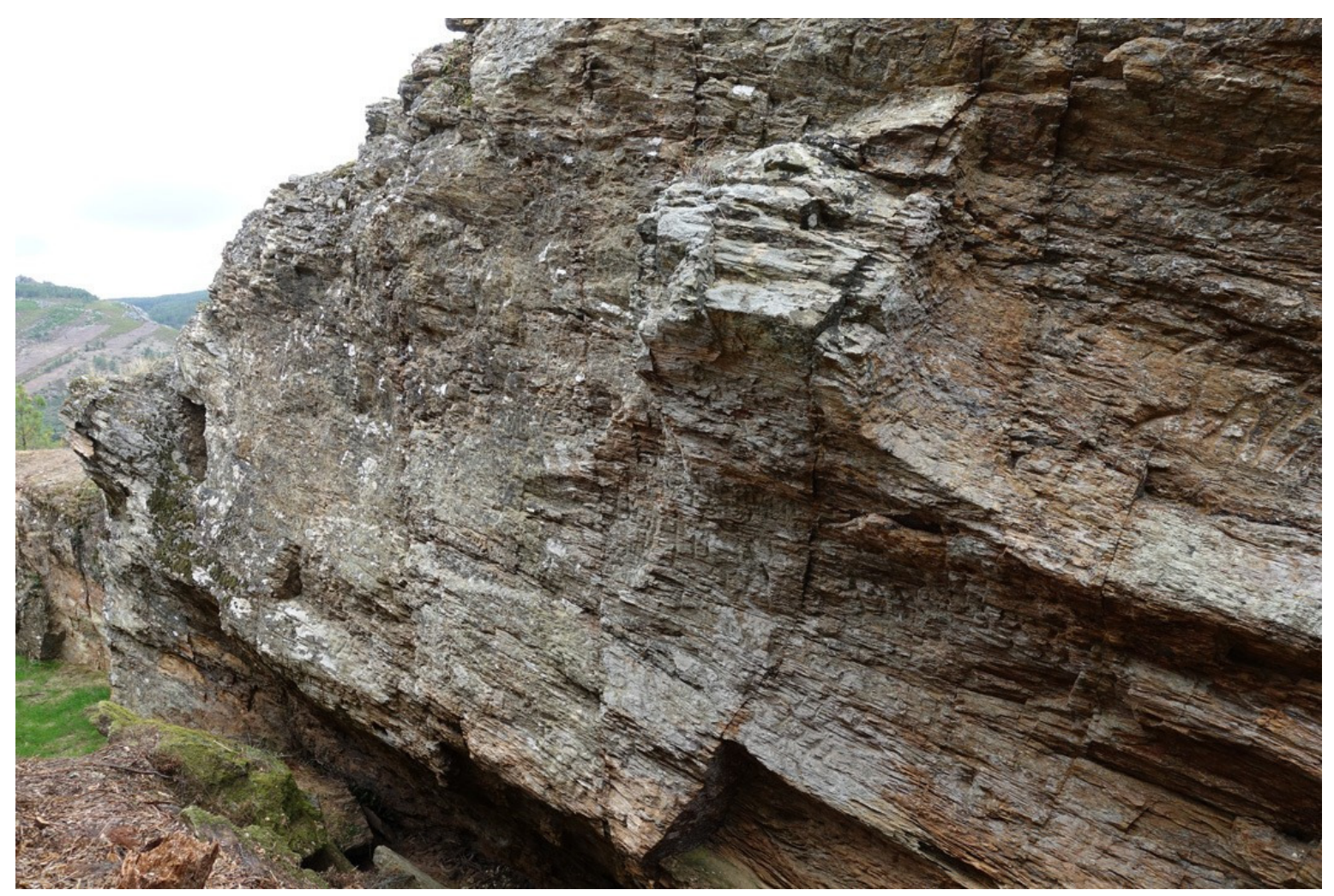

Abb. 6. Bearbeitungsspuren in der nordöstlichen Böschung. Gut erkennbar ist die Begrenzung der Arbeitsspuren nach oben, wo das Hangende eingebrochen ist. In der Mitte des Bildes befindet sich ein abgebrochener Rest, möglicherweise eines Sicherheitsbogens. Links im Bild ist der Durchbruch des Prospektionsschachts (E in Abb. 8) durch die Böschung erkennbar (s. Abb. 9). Foto R. Wahl-Clerici. 


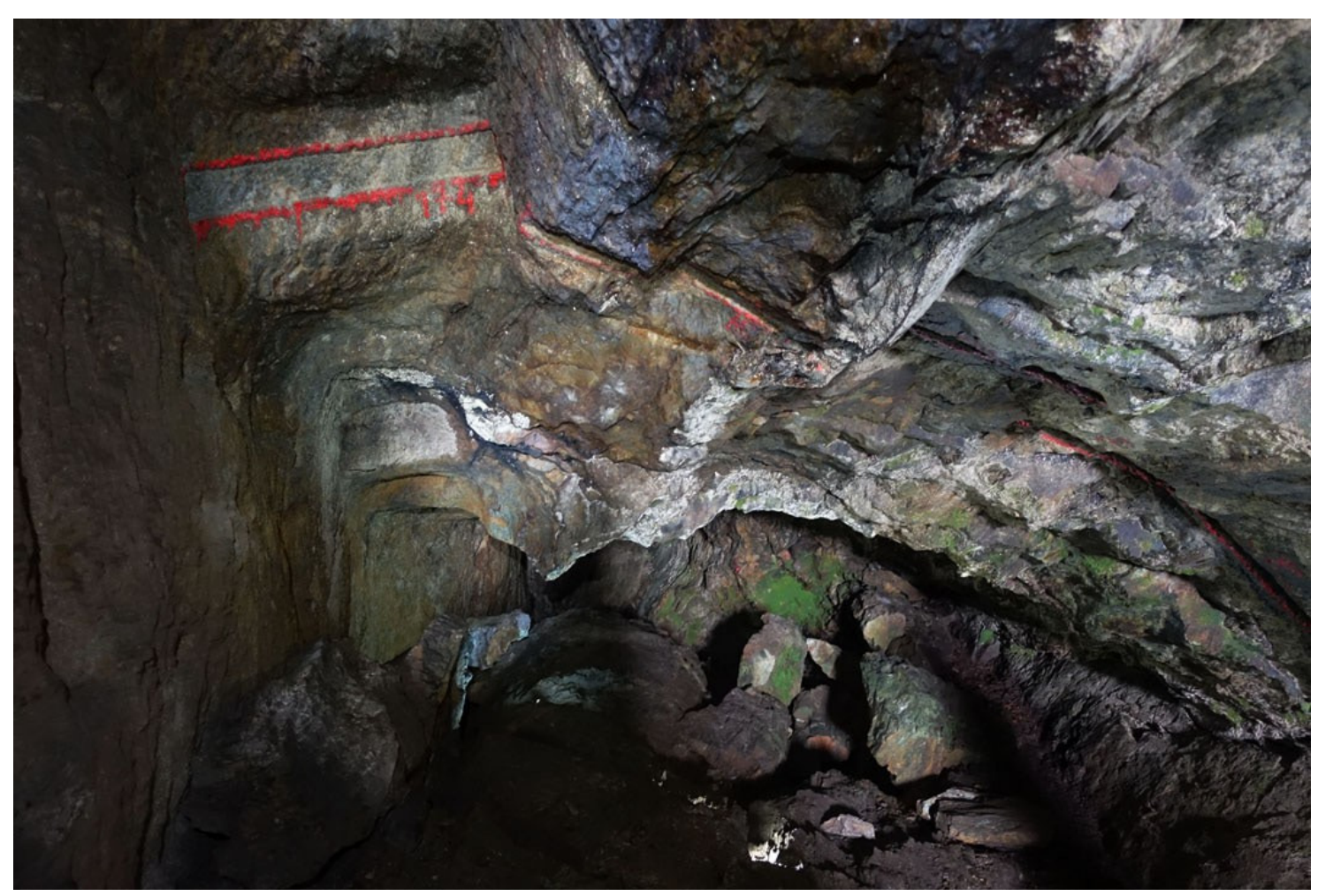

Abb. 7. Blick zum Ende des ohne Hilfsmittel zugängigen Abbaubereichs. Links im Bild ist die Abbauzone, rechts davon der abgebrochene Fels. Markant hebt sich die Probenentnahmestelle 174 hervor. Foto R.Wahl-Clerici.

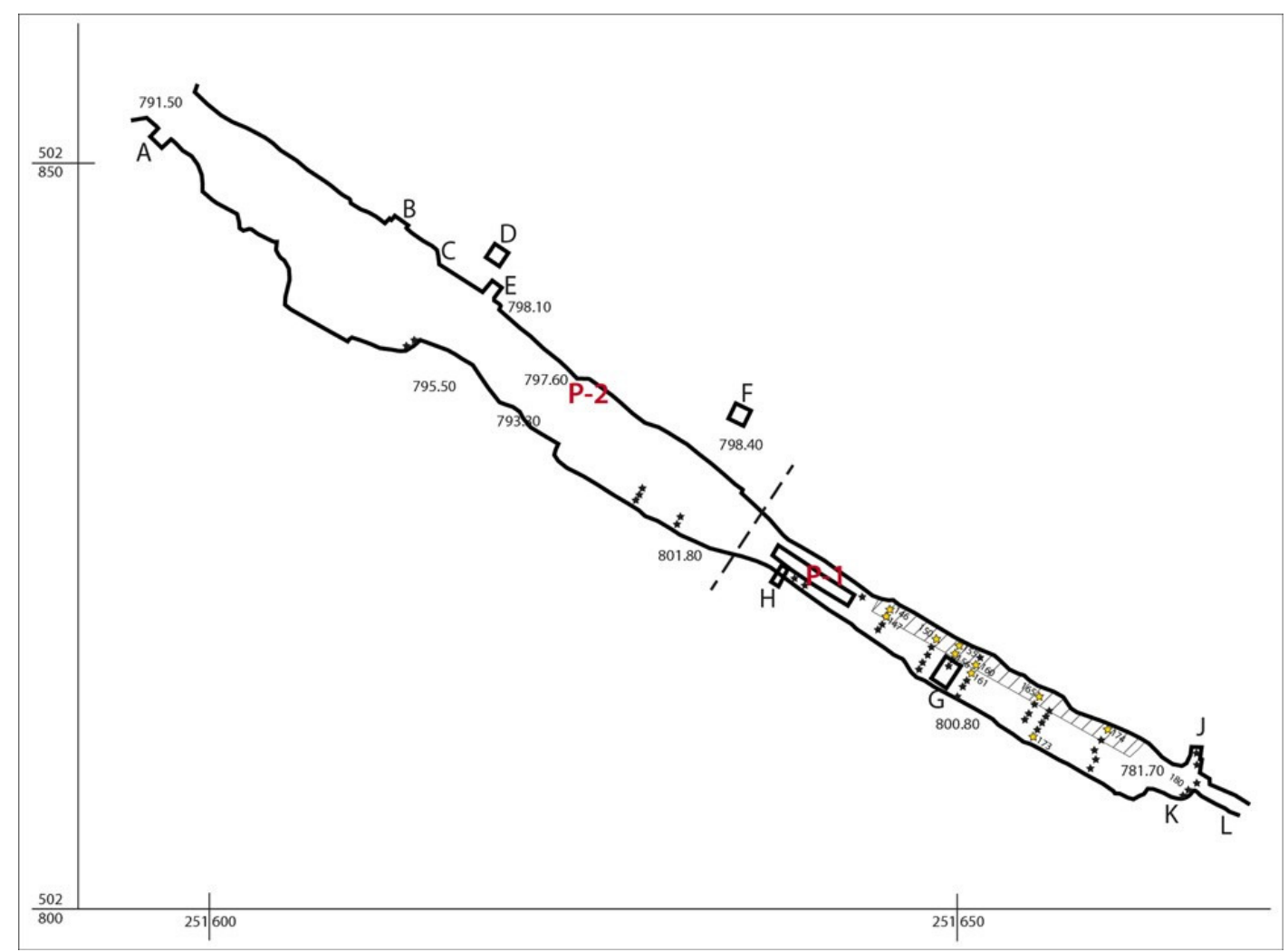

Abb. 8. Grundriss der Abbauzone Lagoinhos. Schraffiert: der römische Abbaubereich. Eingetragen sind die Reste der römischen Prospektionsarbeiten A bis L sowie die P-1 und P-2 (vgl. Abb. 4). Mit Sternchen sind die Probenentnahmestellen markiert, gelb sind die goldhaltigen. Hervorgehoben sind die Proben 147 und 174 aufgrund des hohen Goldgehaltes sowie 180 zur Identifikation der Stellen auf den Abb. 7 und 14. Vorlage Serviço de Fomento Mineiro. Ausführung R. Wahl-Clerici. 
Frankfurter elektronische Rundschau zur Altertumskunde 33 (2017)

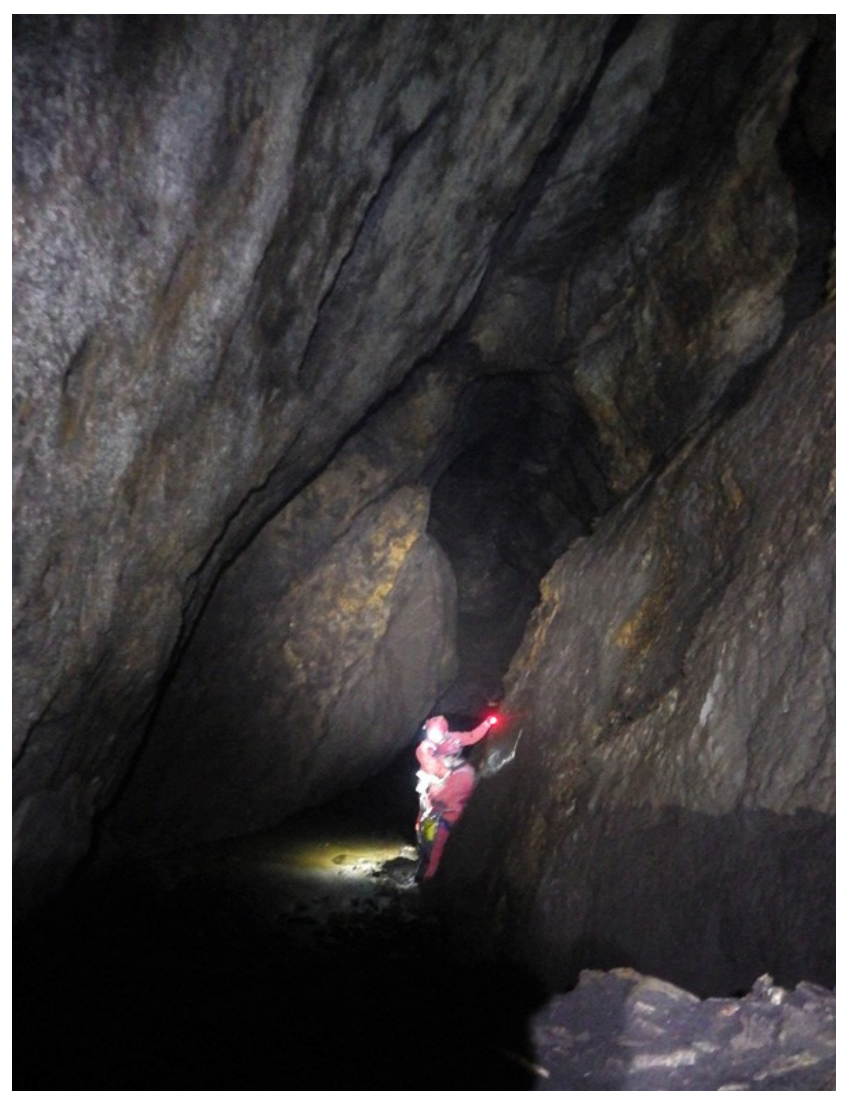

Abb. 9. Blick von NiveauN-3 in Richtung Südost. Ungefähr in der Mittedes Bildes sind noch die ursprünglichen Firste sowie die Ansätze der Stösse eines älteren Niveaus erkennbar. Foto V. Gandra.

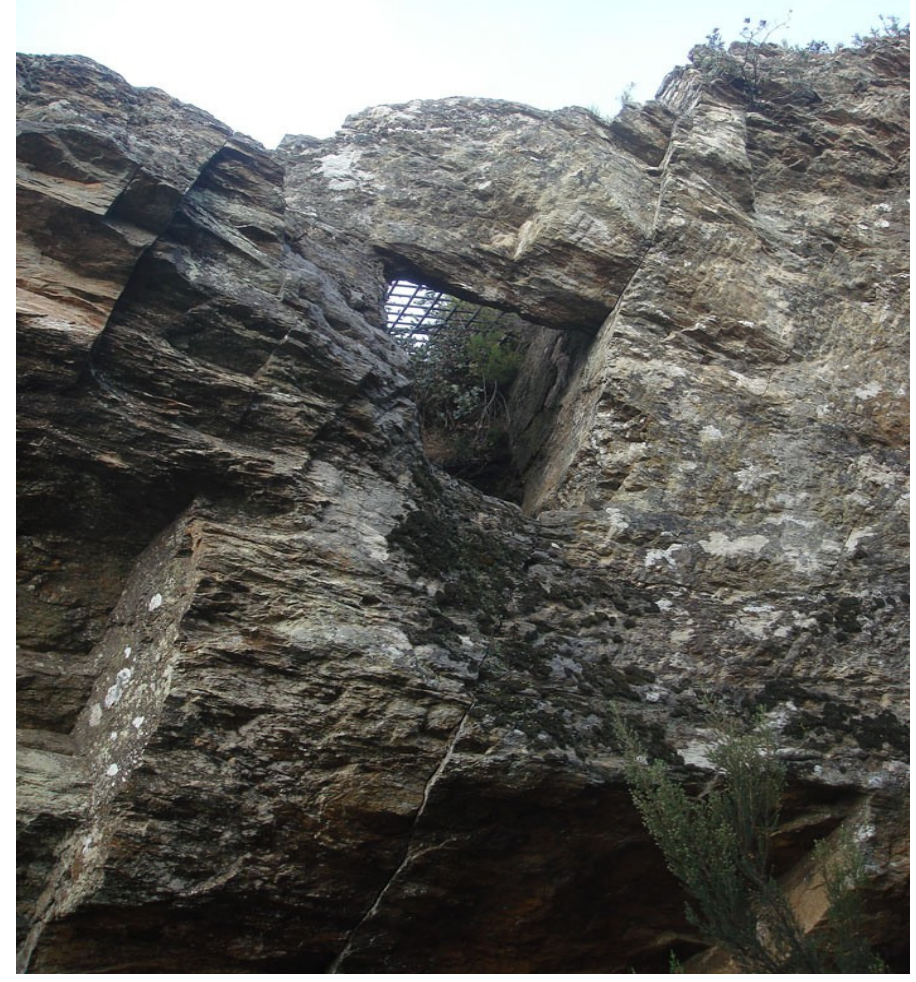

Abb. 10. Durch den Abbau wurde der Prospektionsschacht E durchbrochen. Foto R. Wahl-Clerici. 
Frankfurter elektronische Rundschau zur Altertumskunde 33 (2017)

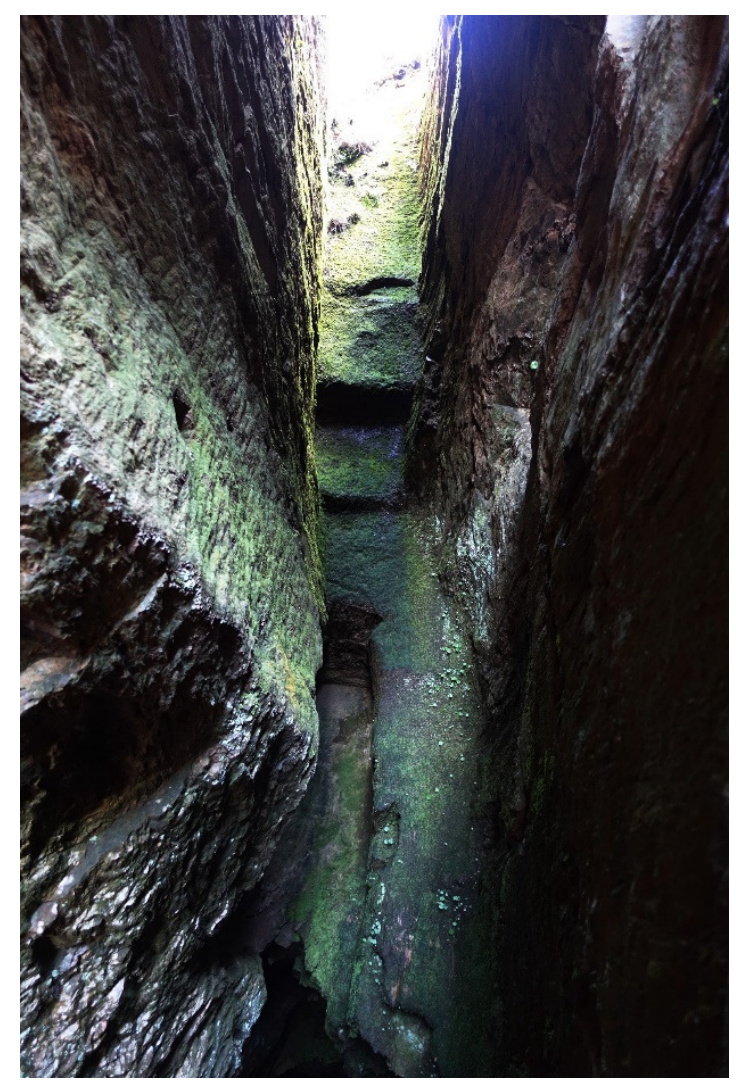

Abb. 11. Blick zum südöstlichen Stoss von Schacht 1 (P-1). Im östlichen Stoss sind die Schlagspuren sowie eine Einarbeitung für die Zimmerung erkennbar. Foto R. Wahl-Clerici.

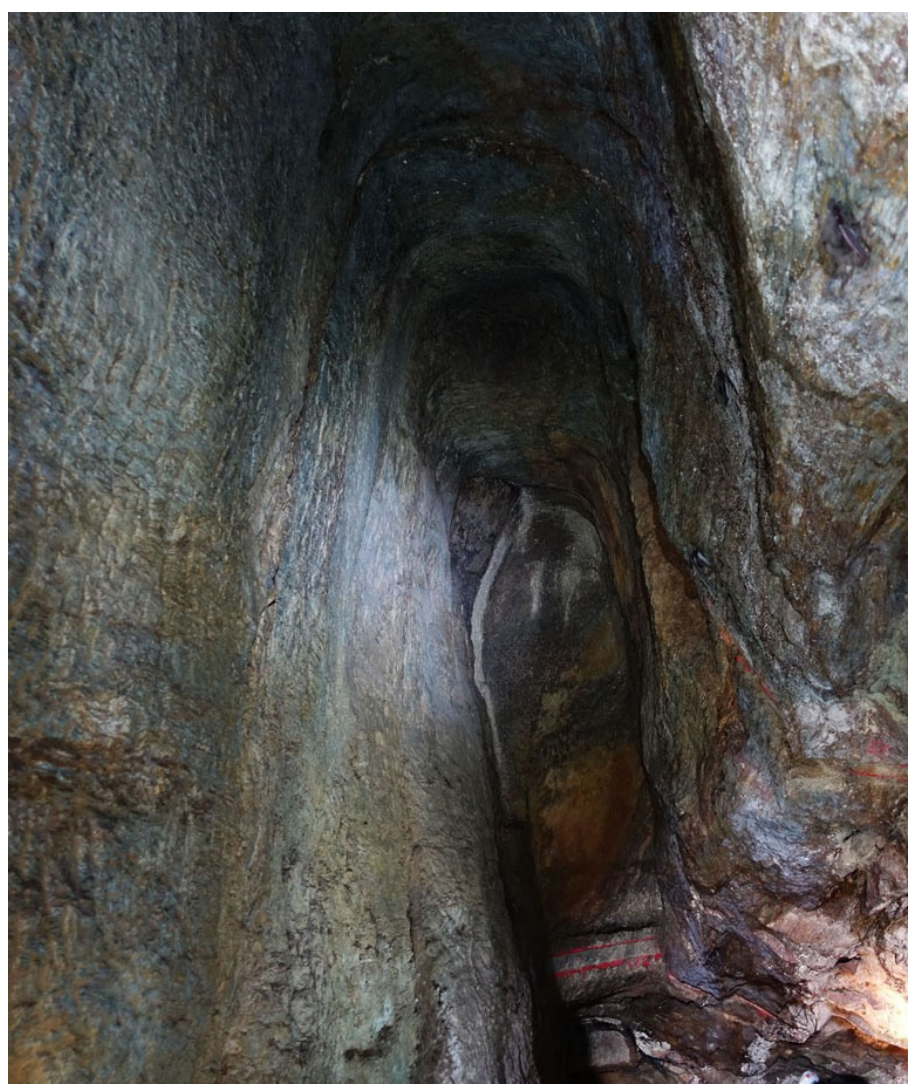

Abb. 12. Blick in Abbauzone im Berginnern von Lagoinhos. Erkennbar ist die Auflassung in Stufen. Aus der tiefsten Stufe wurde die Probe 174 entnommen. Der südöstliche Stoss ist unten weggebrochen (vgl. Abb. 8. 7). Foto 
Frankfurter elektronische Rundschau zur Altertumskunde 33 (2017)

R. Wahl-Clerici.

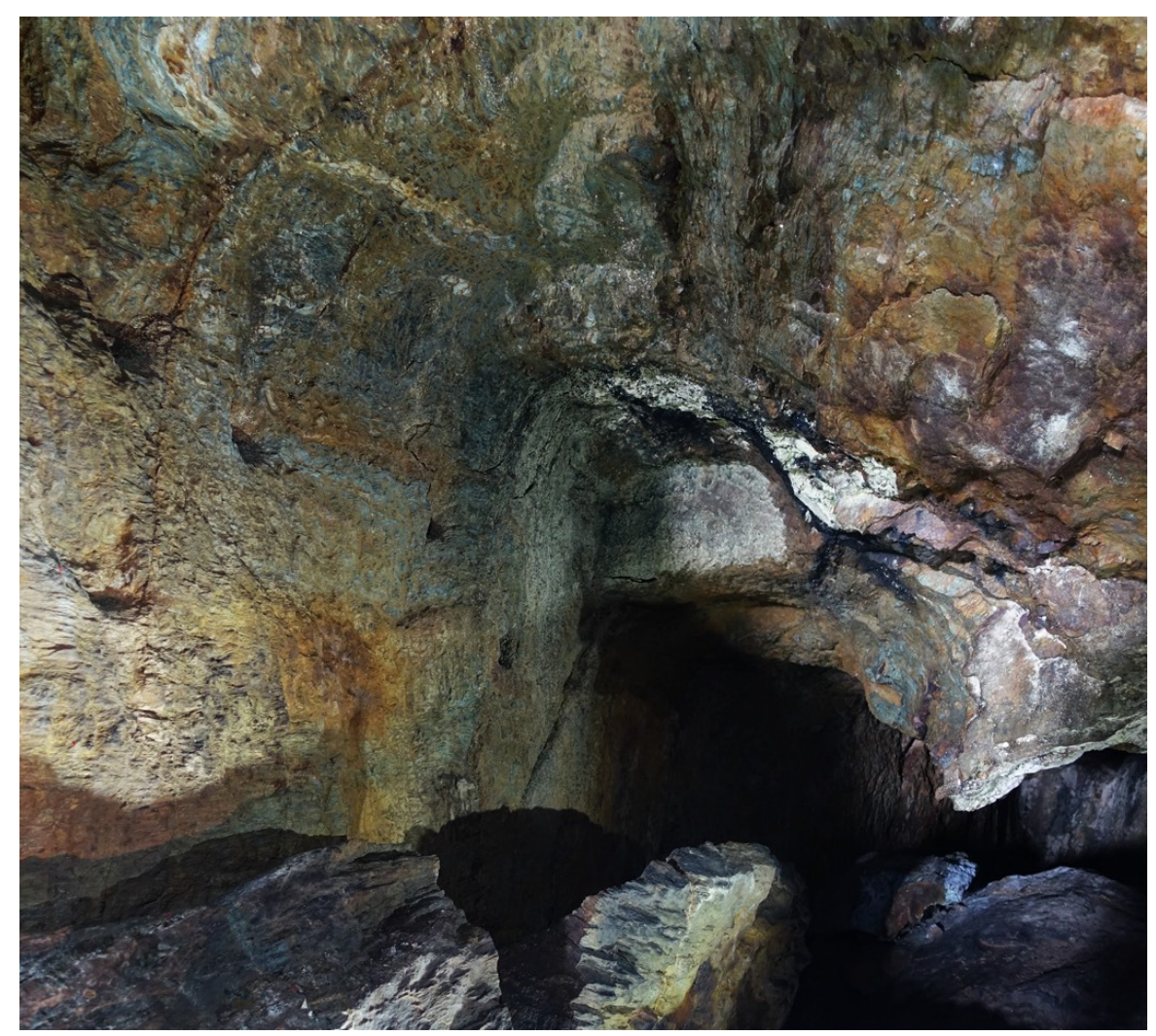

Abb. 13. Blick in das Berginnere mit einer weiteren Abstufung und den Lampennischen im nordöstlichen Stoss. Rechts das abgebrochene Gebirge. Foto R. Wahl-Clerici.

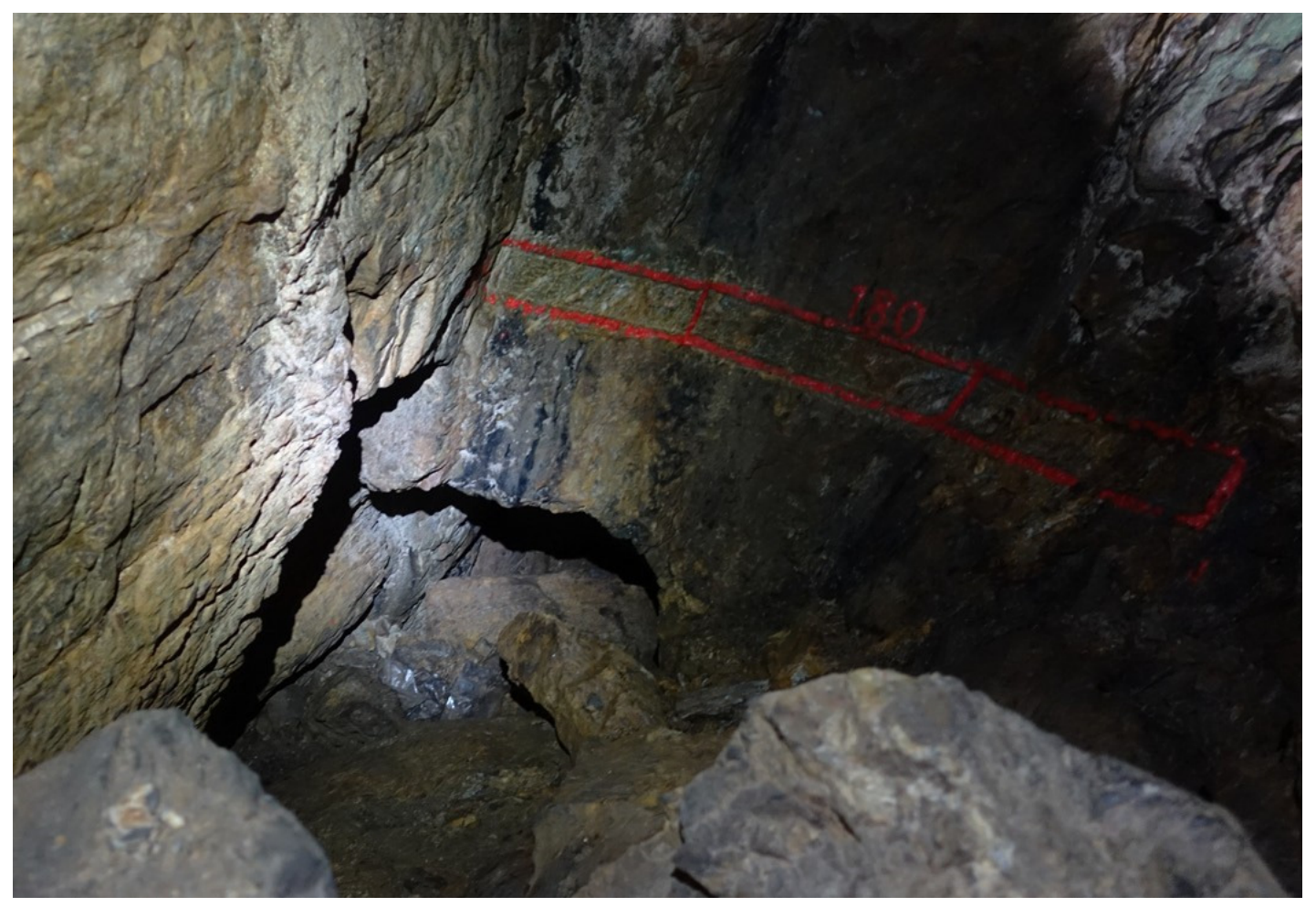

Abb. 14. Blick auf den aufgelassenen Vortrieb mit der sterilen Probe 180. Darunter sindnoch die Prospektionsstrecken PR-2 und PR-4 erkennbar. Foto R. Wahl-Clerici. 
Frankfurter elektronische Rundschau zur Altertumskunde 33 (2017)

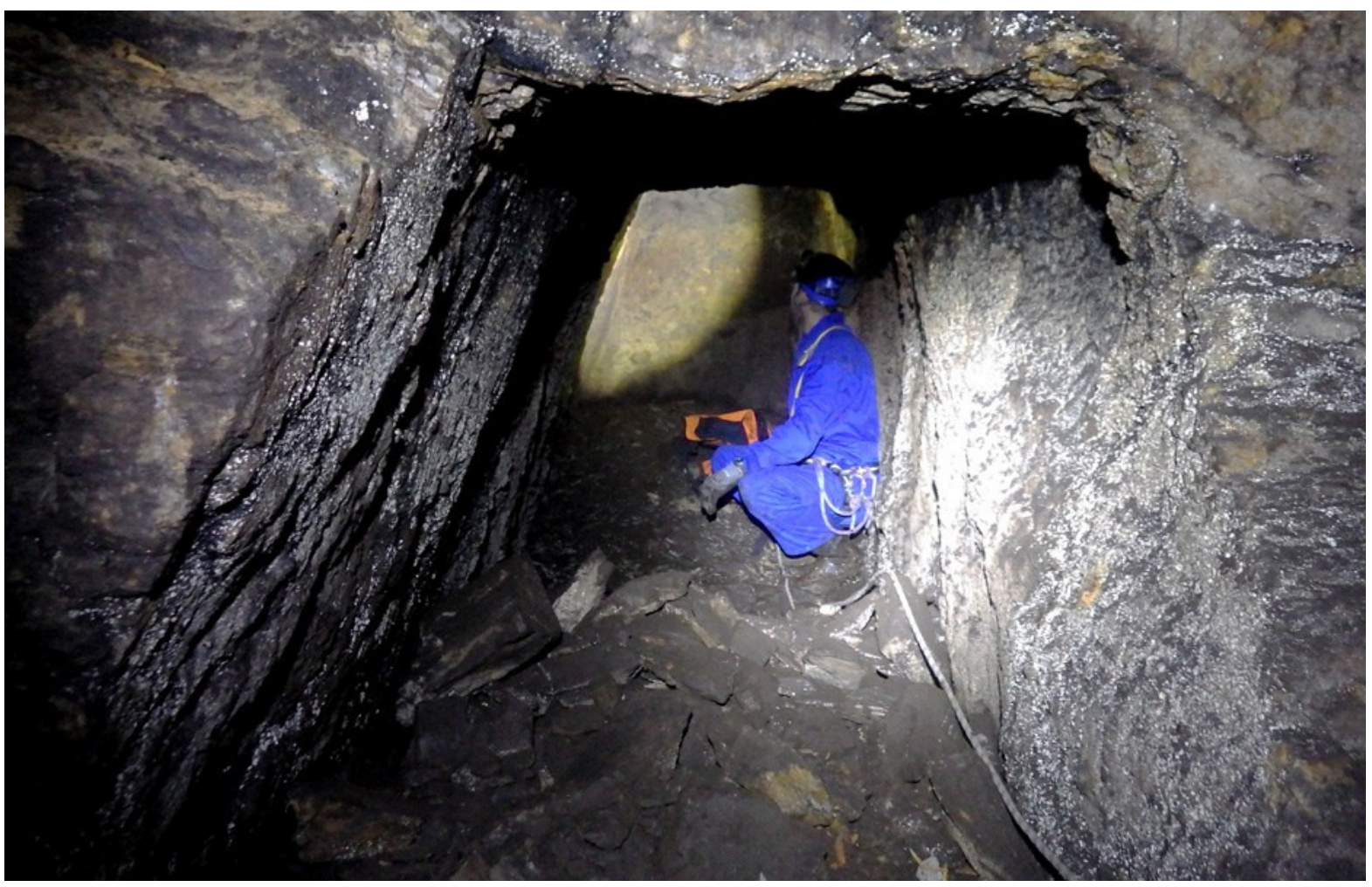

Abb. 15. Blick von Niveau N-2 in die Prospektionsstrecke PR-5. Foto V. Gandra.

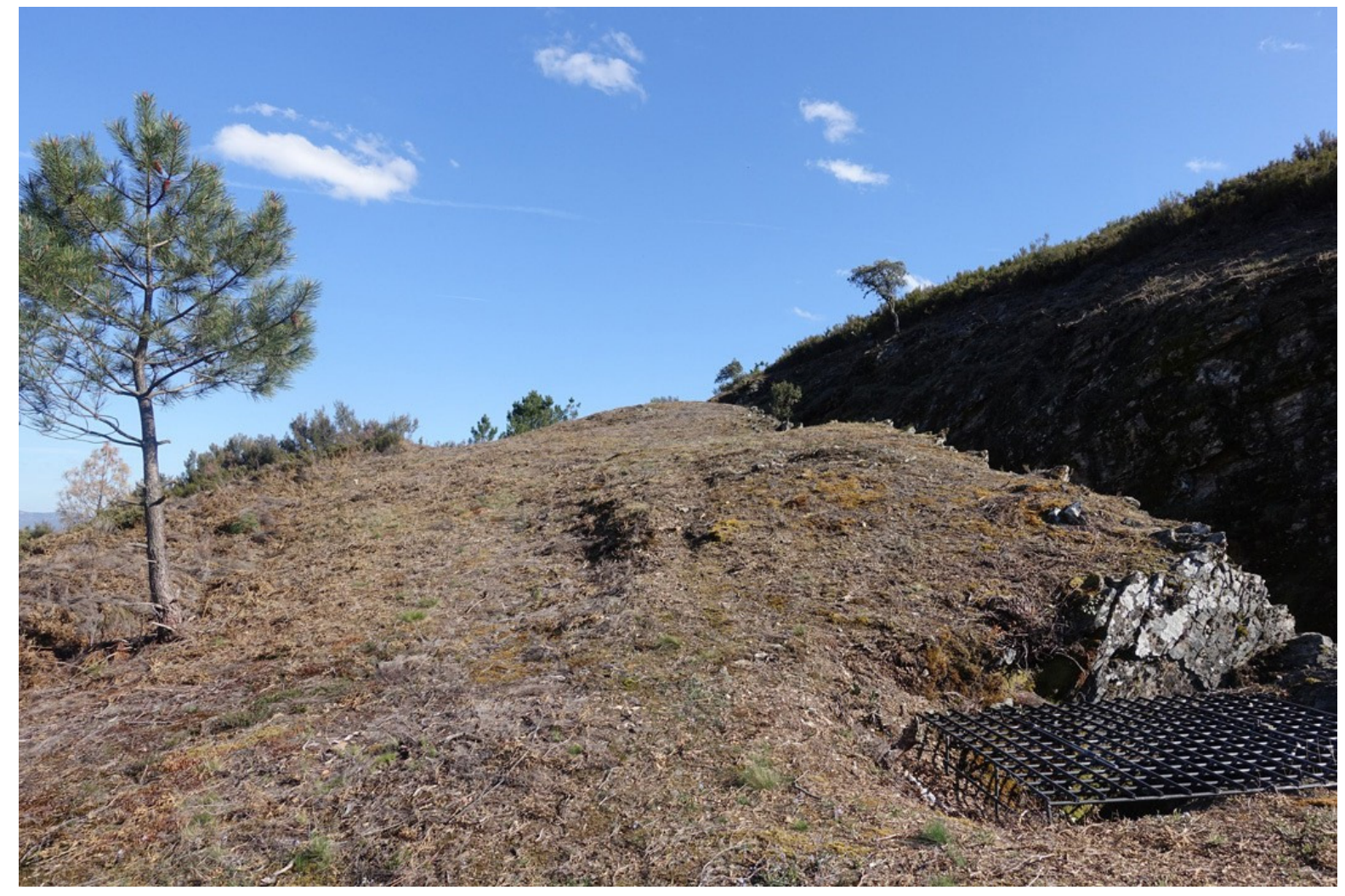

Abb. 16. Blick auf die nordöstlich des Abbaus angehäuften Halden. Von Nordwesten . Foto R. Wahl-Clerici. 
Frankfurter elektronische Rundschau zur Altertumskunde 33 (2017)

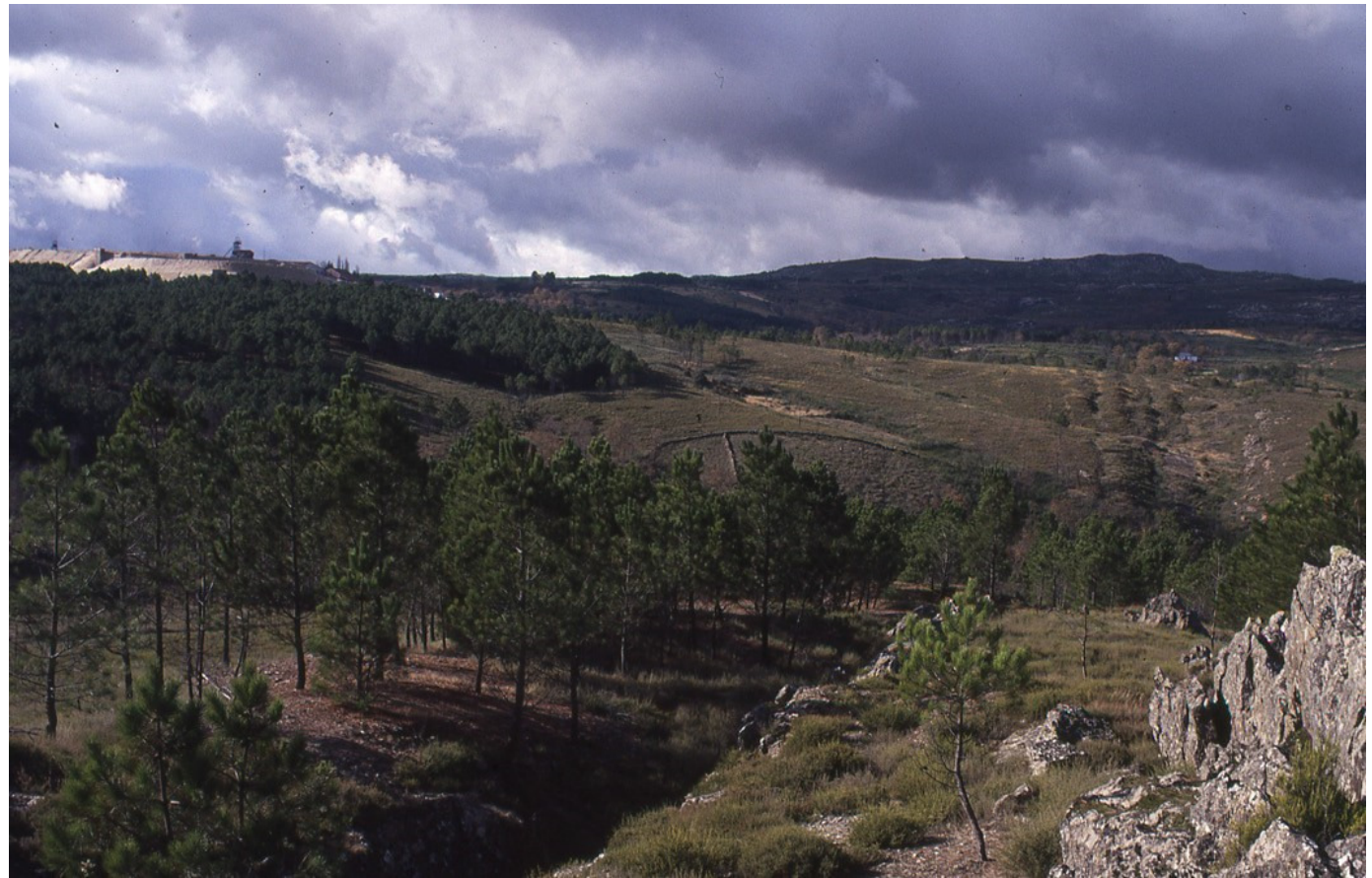

Abb. 17. Blick auf die Abbauzone von Gralheira. Im Vordergrund ist der einfache Graben des Abbaus erkennbar. Dieser fächert sich gegen Westen in mehrere Gräben auf. Links hinten sind die Halden des modernen Abbaus von Jales erkennbar. Von Südsüdost. Foto J. Wahl. 
Frankfurter elektronische Rundschau zur Altertumskunde 33 (2017)

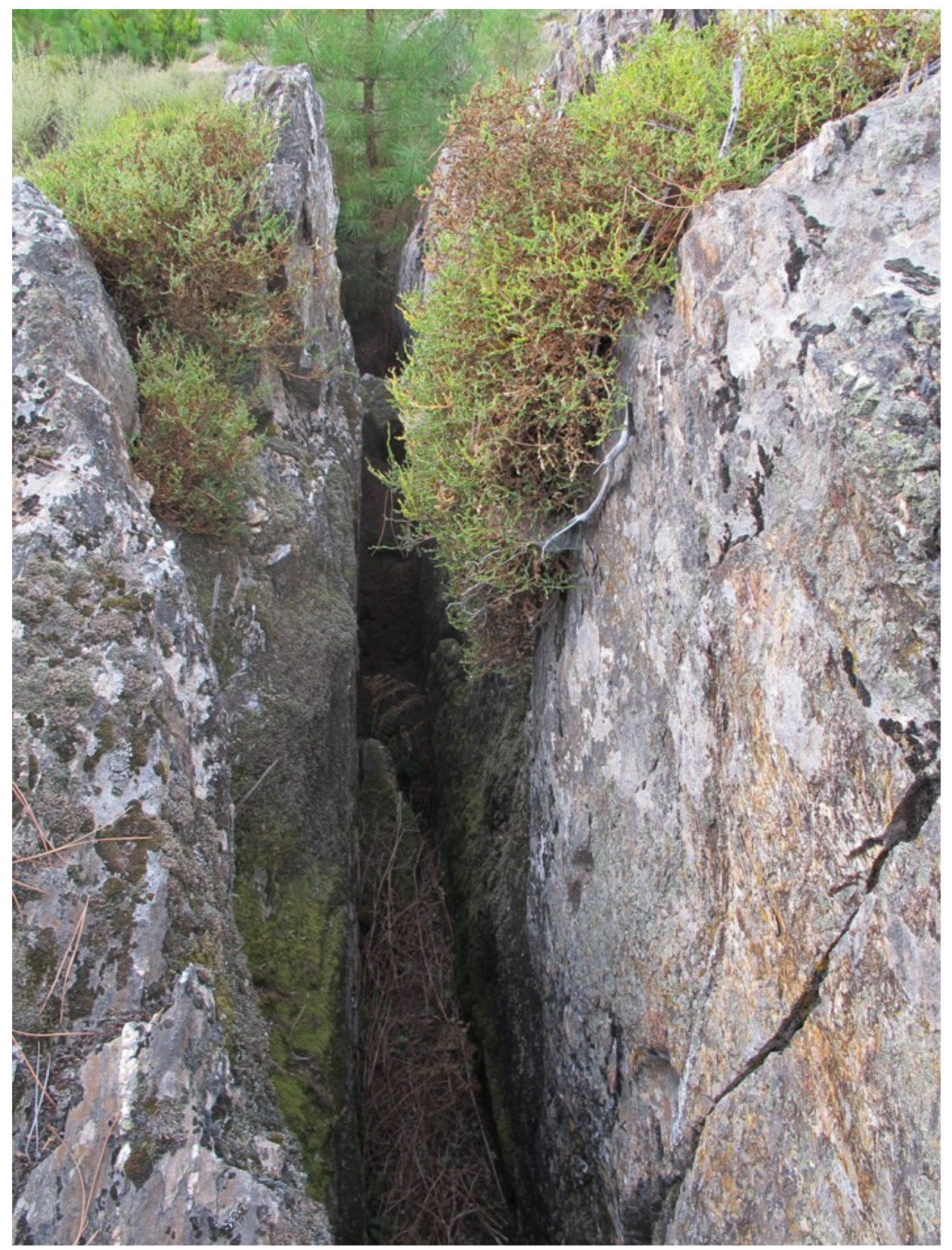

Abb. 18. Gralheira. Obertägiger Abbau mit Einarbeitungen für Spreizung. Von Südsüdost. Foto R. Wahl-Clerici. 